\title{
Jejunal Scarf-Covering Method in Pancreaticojejunostomy after Total Gastrectomy
}

\author{
Takatsugu Oida $^{a}$ Hisao Kano $^{a}$ Kenji Mimatsu $^{a}$ \\ Atsushi Kawasaki $^{\text {a }}$ Youichi Kuboi $^{\text {a }}$ Nobutada Fukino $^{a}$ \\ Kazutoshi Kida ${ }^{a}$ Sadao Amano ${ }^{\text {b }}$ \\ ${ }^{a}$ Department of Surgery, Social Insurance Yokohama Central Hospital, \\ Yokohama, and ${ }^{b}$ Department of Surgery, Nihon University School of Medicine, \\ Tokyo, Japan
}

\section{Key Words}

Pancreaticoduodenectomy · Pancreaticogastrostomy · Pancreaticojejunostomy · Total gastrectomy · Pancreatic fistula $\cdot$ Jejunal loop

\begin{abstract}
Pancreatic fistula is the most serious postoperative complication after pancreaticoduodenectomy, and it leads to intra-abdominal abscess, sepsis, hemorrhage and high mortality. To prevent pancreatic fistula, wrapping of skeletonized vessels and the anastomotic site of the pancreaticoenterostomy using the round ligament, greater omentum, or both has been evaluated. However, the round ligament and greater omentum have already been resected in patients who have previously undergone total gastrectomy, making them unavailable in pancreaticoduodenectomy. Therefore, we developed a procedure for wrapping the anastomotic site of the pancreaticojejunostomy using the jejunum, namely the 'jejunal scarf-covering method' as a novel technique to prevent pancreatic fistula following pancreaticoduodenectomy in patients who have previously undergone total gastrectomy.
\end{abstract}

\section{Introduction}

Pancreaticoduodenectomy (PD) is commonly used for the surgical treatment of malignancies of the ampulla of Vater, duodenum, head of the pancreas and distal common bile duct. Recent advances in surgical techniques and perioperative management have resulted in low mortality rates; however, the morbidity rate after PD remains high. Pancreatic fistula (PF), in particular, is the most serious postoperative complication $[1,2]$. PF leads to intra-abdominal abscess, sepsis, hemorrhage and high 
mortality [3, 4]. To prevent PF, wrapping of skeletonized vessels and the anastomotic site of the pancreaticoenterostomy using the round ligament $[5,6]$, greater omentum $[7,8]$, or both [9] has been evaluated. With the increase in life expectancy in recent years, the probability of patients having to undergo a second operation for a different diagnosis from that of the original operation has increased. Consequently, there is an increased probability of performing PD in patients with pancreatic or periampullary cancer who have previously undergone gastrectomy. When PD is performed as a second operation, it is important to consider the gastroenteric reconstruction used in the prior operation when selecting an appropriate reconstruction following PD. However, the round ligament and the greater omentum have been resected in patients who have already undergone gastrectomy, making their use unavailable following PD. Thus, we developed a method of wrapping of the anastomotic site of the pancreaticojejunostomy (PJ) using the jejunum. This 'jejunal scarf-covering method' is a novel technique that can prevent PF following PD for patients who have previously undergone total gastrectomy.

\section{Case Report}

A 68-year-old man underwent left hemicolectomy due to descending colon cancer 15 years ago, and a total gastrectomy due to gastric cancer 4 years ago. Follow-up contrast-enhanced computed tomography in an outpatient clinic revealed bile duct dilation and a palpable gallbladder but no jaundice. On admission, laboratory studies indicated increased levels of aspartate aminotransferase (59 IU/l), alanine aminotransferase (166 IU/l) and alkaline phosphatase (999 IU/l); however, the levels of serum total bilirubin $(0.7 \mathrm{mg} / \mathrm{dl})$ and tumor markers such as carbohydrate antigen 19-9 (37 U/ml), carcinoembryonic antigen $(0.9 \mathrm{ng} / \mathrm{ml})$, DUPAN-2 $(25 \mathrm{U} / \mathrm{ml})$ and Span-1 (19 U/ml) were normal. Abdominal ultrasonography revealed a heterogeneous hypoechoic mass in the common bile duct and markedly dilated bile ducts over the neoplasm. Computed tomography did not reveal any metastatic tumors in the liver or the intra-abdominal cavity. Magnetic resonance cholangiopancreatography indicated dilation of the upper common bile duct and biliary tree as well as tumor defection at the lower common bile duct (fig. 1). The pancreatic duct was not dilated. Contrast-enhanced arterial and portal phase images showed no encasement of the artery or portal vein. From these results, adenocarcinoma of the common bile duct was diagnosed; therefore, we performed PD.

The original operations involved left hemicolectomy with end-to-end transverse colonrectosigmoid anastomosis and total gastrectomy with Roux-en-Y reconstruction. In this case, the colon was displaced toward the right side of the abdomen and the Roux limb was $40 \mathrm{~cm}$ from the ligament of Treitz.

The second portion of the duodenum was resected $20 \mathrm{~cm}$ proximal from the ligament of Treitz with resection of the gallbladder and common bile duct, as well as removal of the pancreas head. A pancreatic stent was inserted into and fixed within the pancreatic duct. The proximal jejunum was brought to the hepatic hilum in the retrocolic position. The proximal jejunum was $15 \mathrm{~cm}$ long and bent downward, and the serosa of the jejunum was then sutured side-to-side for 5-6 cm using 3-0 silk to create a double jejunal loop. The remnant pancreas with the pancreatic duct tube was placed on the jejunal double loop. A bilayer end-to-side anastomosis was constructed using duct-to-mucosa anastomosis with interrupted 4-0 absorbable sutures reinforced by a seromuscular-parenchymal layer of 3-0 silk at one side of the jejunum on the jejunal double loop. The pancreatic duct tube was brought out through the jejunum and the abdominal wall (fig. 2a). After PJ, the proximal site of the jejunal loop that was opposite the PJ was bent over, rolled around and wrapped around the anastomosis of the PJ (fig. 2b). The area surrounding the serosa of the jejunum was sutured to the pancreatic parenchyma using 3-0 silk (fig. 2c). Finally, an end-to-side hepaticojejunostomy was performed using a single layer of interrupted 4-0 absorbable monofilament sutures and an inserted biliary lost tube (fig. 3). Two drains were placed (one for the hepaticojejunostomy, the other for the PJ) and brought out on the right just below the subcostal incision. 
Operative time was $7 \mathrm{~h} 25 \mathrm{~min}$, blood loss was 1,430 ml, and 4 units of blood were required. According to the general rules of the surgical and pathological studies on cancer of the biliary tract proposed by the Japanese Society of Biliary Surgery, this tumor was a moderately differentiated tubular adenocarcinoma of the bile duct and was classified as a stage VIa tumor $\left(\mathrm{pT}_{4}, \mathrm{H}_{0}, \mathrm{P}_{0}, \mathrm{M}-\right)$. $\mathrm{PF}$ did not develop in this patient. One month after surgery, the patient's serum albumin level was normal $(3.9 \mathrm{~g} / \mathrm{dl})$ and his weight had returned to that before the operation.

\section{Discussion}

Major pancreatic resection currently provides the only possibility of cure, or even increased survival, in patients with pancreatic and periampullary cancers. Consequently, there is an increased probability that patients with pancreatic or periampullary cancer who have previously undergone gastrectomy will undergo PD. However, PF has been the major concern in PD. Several studies have shown that pancreaticogastrostomy is associated with a very low rate of anastomotic leakage, suggesting that it is safer than PJ $[10,11]$. However, pancreaticogastrostomy is theoretically unsuitable because the remnant stomach is small after gastrectomy, particularly in patients who have previously undergone distal or extensive gastrectomy. Thus, $\mathrm{PJ}$ is performed as pancreaticoenterostomy in patients who have previously undergone total gastrectomy.

To prevent PF, wrapping of skeletonized vessels and the anastomotic site of the pancreaticoenterostomy using the round ligament $[5,6]$, greater omentum $[7,8]$, or both [9] has been evaluated. In general, Roux-en-Y reconstruction is performed after total gastrectomy, and the round ligament and the greater omentum are subsequently resected during total gastrectomy. Thus, wrapping the anastomotic site of pancreaticoenterostomy using the round ligament or the greater omentum is not possible.

It has been proposed that a soft, friable, and normal pancreas of normal size with a thin-walled main pancreatic duct increases the risk of PF. Nevertheless, surgical technique still plays a crucial role in preventing PF. Therefore, we routinely use a pancreatic stent tube to drain external pancreatic juice. Moreover, we use a jejunum substitute for the round ligament or the greater omentum to wrap the pancreatic anastomotic site.

After total gastrectomy, malnutrition and weight loss due to absence of gastric function are major issues. Further invasive surgeries such as PD may increase these nutritional deficiencies. Duodenum-preserving resection of the head of the pancreas was first introduced by Beger et al. [12] for chronic pancreatitis. Since then, several modified procedures have been introduced, including pancreatic head resection with second-portion duodenectomy (PHRSD) [13, 14]. However, Ahn et al. [15] found that preservation of the duodenal segment may play a significant role in the absorption of iron, calcium, fat and folic acid in PHRSD. The duodenum and the upper part of the jejunum are very important portions with regard to gut hormone secretion. Thus, preservation of the duodenal segment and the upper jejunum may be desirable for prevention of malnutrition. Fortunately, in our case, the Roux limb was longer and we were able to preserve the third portion of the duodenum using the Roux limb longitudinally in an effort to maintain gut hormone secretion. Moreover, this technique 
does not require jejunojejunostomy and reduces the anastomosis. As a result, the patient's nutritional state was fairly maintained.

To our knowledge, there have been no reports on a similar jejunal scarf-covering method to prevent PF following PD in patients who have undergone total gastrectomy. This constructive technique might be useful for preventing PF and maintaining postoperative nutrition in patients who have previously undergone total gastrectomy.

\section{Disclosure Statement}

The authors did not have any financial grants or funding in this study.

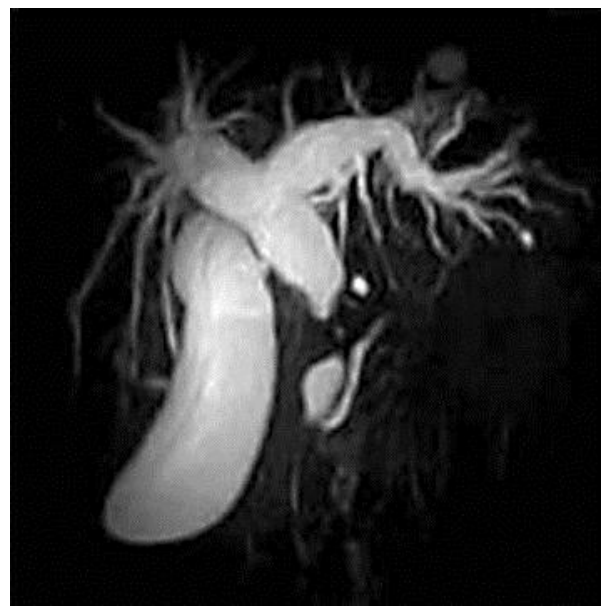

Fig. 1. Preoperative magnetic resonance cholangiopancreatography revealed dilation of the upper common bile duct and biliary tree as well as tumor defection at the lower common bile duct. 


\begin{tabular}{r|l|l|l} 
Case Reports in & $\begin{array}{l}\text { Case Rep Gastroenterol 2012;6:472-477 } \\
\text { DOI: 10.1159/000341520 }\end{array}$ & $\begin{array}{l}\text { Published online: } \\
\text { July 19, 2012 }\end{array}$ & $\begin{array}{l}\text { @ 2012 S. Karger AG, Basel } \\
\text { ISSN 1662-0631 } \\
\text { www.karger.com/crg }\end{array}$ \\
\hline
\end{tabular}
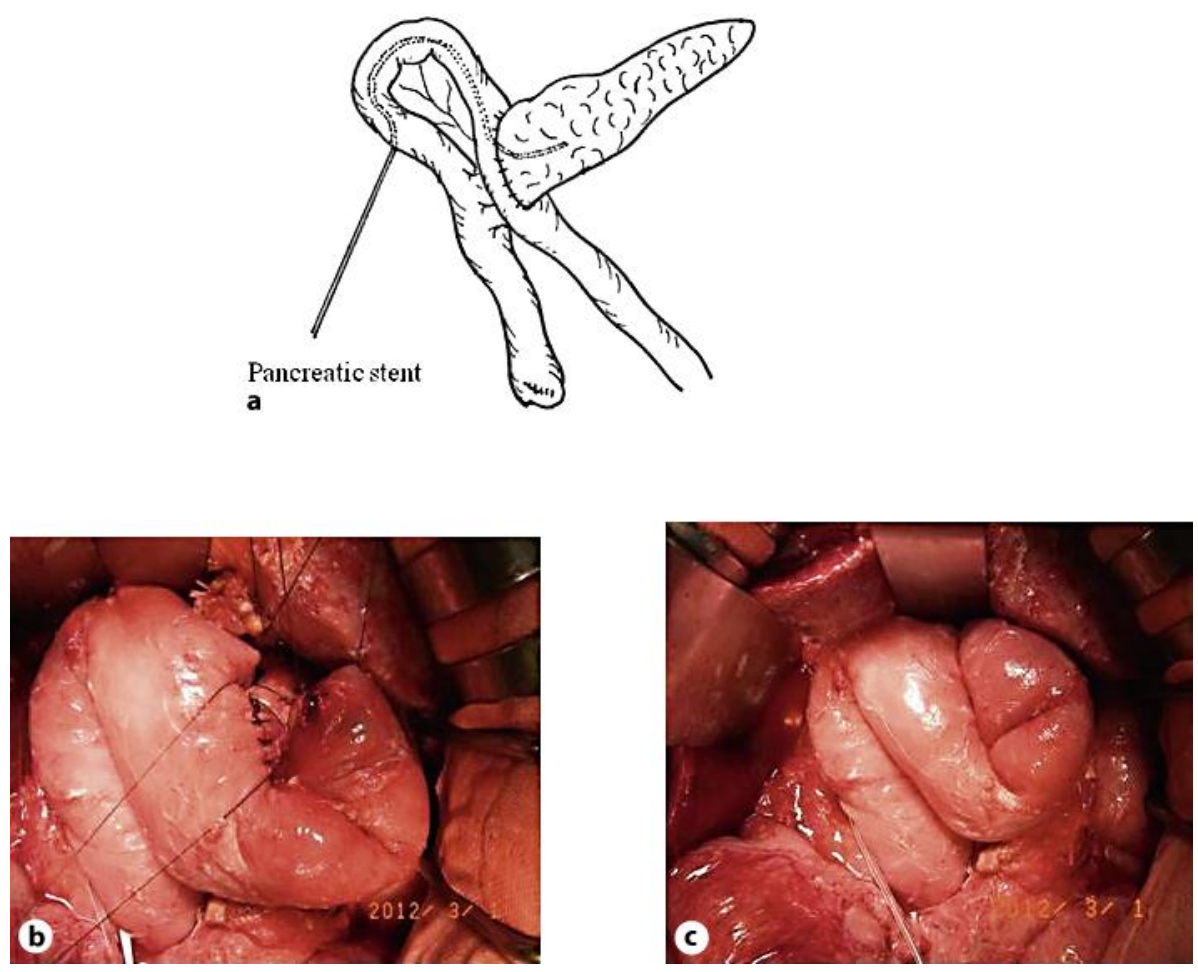

Fig. 2. Covering jejunal double loop. The proximal jejunum was brought to the hepatic hilum in the retrocolic position. a The proximal jejunum was $15 \mathrm{~cm}$ long and bent downward, and the serosa of the jejunum was then sutured side-to-side for 5-6 cm using 3-0 silk to create a double jejunal loop. b The proximal site of the jejunal double loop opposite the PJ was bent over, rolled around and wrapped around the anastomosis of the PJ. c The area surrounding the serosa of the jejunum was sutured to the pancreatic parenchyma using 3-0 silk.

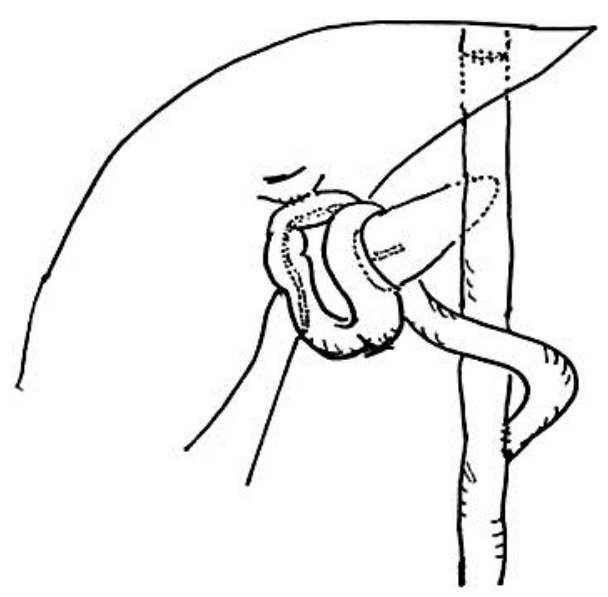

Fig. 3. Reconstruction findings. An end-to-side hepaticojejunostomy was performed using a single layer of interrupted 4-0 absorbable monofilament sutures and an inserted biliary lost tube. 


\section{References}

1 Cullen JJ, Sarr MG, Ilstrup DM: Pancreatic anastomotic leak after pancreaticoduodenectomy: incidence, significance, and management. Am J Surg 1994;168:295-298.

-2 Yeo CJ, Cameron JL, Sohn TA, Lillemoe KD, Pitt HA, Talamini MA, Hruban RH, Ord SE, Sauter PK, Coleman J, Zahurak ML, Grochow LB, Abrams RA: Six hundred fifty consecutive pancreaticoduodenectomies in the 1990s: pathology, complications, and outcomes. Ann Surg 1997;226:248-260.

3 Trede M, Schwall G: The complications of pancreatectomy. Ann Surg 1988;207:39-47.

-4 Miedema BW, Sarr MG, van Heerden JA, Nagorney DM, McIlrath DC, Ilstrup D: Complications following pancreaticoduodenectomy: current management. Arch Surg 1992;127:945-950.

5 Sakamoto Y, Shimada K, Esaki M, Kajiwara T, Sano T, Kosuge T: Wrapping the stump of the gastroduodenal artery using the falciform ligament during pancreaticoduodenectomy. J Am Coll Surg 2007;204:334-336.

6 Abe N, Sugiyama K, Suzuki Y, Yanagida O, Masaki T, Mori T, Atomi Y: Falciform ligament in pancreaticoduodenectomy for protection of skeletonized and divided vessels. J Hepatobiliary Pancreat Surg 2009;16:184-188.

7 Kurosaki I, Hatakeyama K: Omental wrapping of skeletonized major vessels after pancreaticoduodenectomy. Int Surg 2004;89:90-94.

$>8$ Maeda A, Ebata T, Kanemoto H, Matsunaga K, Bando E, Yamaguchi S, Uesaka K: Omental flap in pancreaticoduodenectomy for protection of splanchnic vessels. World J Surg 2005;29:1122-1126.

-9 Mimatsu K, Oida T, Kano H, Kawasaki A, Fukino N, Kida K, Kuboi Y, Amano S: Protection of major vessels and pancreaticogastrostomy using the falciform ligament and greater omentum for preventing pancreatic fistula in soft pancreatic texture after pancreaticoduodenectomy. Hepatogastroenterology 2011;58:1782-1786.

10 Miyagawa S, Makuuchi M, Lygidakis NJ, Noguchi T, Nishimaki K, Hashikura Y, Harada H, Hayashi K, Kakazu T: A retrospective comparative study of reconstructive methods following pancreaticoduodenectomy-pancreaticojejunostomy versus pancreaticogastrostomy. Hepatogastroenterology 1992;39:381-384

11 Takano S, Ito Y, Watanabe Y, Yokoyama T, Kubota N, Iwai S: Pancreaticogastrostomy versus pancreaticogastrostomy in reconstruction following pancreaticoduodenectomy. Br J Surg 2000;87: 423-427.

12 Beger HG, Buchler M, Bittner R, Uhl W: Duodenum-preserving resection of the head of the pancreas - an alternative to Whipple's procedure in chronic pancreatitis. Hepatogastroenterology 1990;37:283-289.

13 Kim SW, Jang JY, Park SJ, Park YC, Park YH: Pancreatic head resection with segmental duodenectomy. J Korean Surg Soc 2000;59:519-525.

14 Isaji S, Kawarada Y: Pancreatic head resection with second-portion duodenectomy for benign lesions, low-grade malignancies, and early stage carcinomas involving the pancreatic head region. Am J Surg 2001;181:172-176.

15 Ahn YJ, Kim SW, Park YC, Jang JY, Yoon YS, Park YH: Duodenal-preserving resection of the head of the pancreas and pancreatic head resection with second-portion duodenectomy for benign lesions, low-grade malignancies, and early carcinoma involving the periampullary region. Arch Surg 2003;138: 162-168. 